\title{
New contiguity relation of the sixth Painlevé equation from a truncation *
}

\author{
Robert Conte† and Micheline Musette $\ddagger$ \\ †Service de physique de l'état condensé, CEA-Saclay \\ F-91191 Gif-sur-Yvette Cedex, France \\ E-mail: Conte@drecam.saclay.cea.fr \\ $\ddagger$ Dienst Theoretische Natuurkunde, Vrije Universiteit Brussel \\ Pleinlaan 2, B-1050 Brussels, Belgium \\ E-mail: MMusette@vub.ac.be
}

Submitted February 2001, accepted 24 October 2001

\begin{abstract}
For the master Painlevé equation P6(u), we define a consistent method, adapted from the Weiss truncation for partial differential equations, which allows us to obtain the first degree birational transformation of Okamoto. Two new features are implemented to achieve this result. The first one is the homography between the derivative of the solution $u$ and a Riccati pseudopotential. The second one is an improvement of a conjecture by Fokas and Ablowitz on the structure of this birational transformation. We then build the contiguity relation of P6, which yields one new second order nonautonomous discrete equation.
\end{abstract}

Keywords: Painlevé equations, birational transformation, contiguity relation, Schlesinger transformation, singular manifold method, truncation.

PACS 02.30.+g

\section{Contents}

\begin{tabular}{lll}
\hline 1 & Introduction & 2
\end{tabular}

2 The fundamental homography $\quad 4$

$\begin{array}{lll}3 & \text { The truncation } & 5\end{array}$

\begin{tabular}{|lll}
\hline 4 & The elementary birational transformation of P6 & 6
\end{tabular}

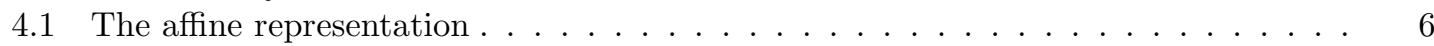

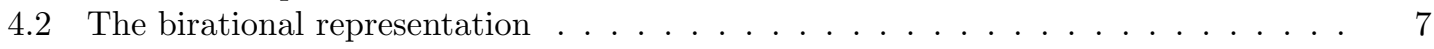

\begin{tabular}{lll}
\hline 5 & Contiguity relation & 7
\end{tabular}

$\begin{array}{lll}6 & \text { On Schlesinger transformations } & 9\end{array}$

$\begin{array}{lll}7 \text { Conclusion } & 10\end{array}$

8 Appendix. One-family truncation for P6 10

*To appear in Physica D. Preprint S2001/009. Corresponding author RC, e-mail Conte@drecam.saclay.cea.fr, phone $+33-1-69087349$, fax $+33-1-69088786$. 


\section{Introduction}

Second order first degree algebraic ordinary differential equations (ODEs) define six and only six functions [1], which satisfy neither a first order ODE nor a linear ODE. Since the sixth of these Painlevé equations Pn can generate the five others by a confluence process [2], we will mainly concentrate on it

$$
\begin{aligned}
\text { P6 : } u^{\prime \prime}= & \frac{1}{2}\left[\frac{1}{u}+\frac{1}{u-1}+\frac{1}{u-x}\right] u^{\prime 2}-\left[\frac{1}{x}+\frac{1}{x-1}+\frac{1}{u-x}\right] u^{\prime} \\
& +\frac{u(u-1)(u-x)}{x^{2}(x-1)^{2}}\left[\alpha+\beta \frac{x}{u^{2}}+\gamma \frac{x-1}{(u-1)^{2}}+\delta \frac{x(x-1)}{(u-x)^{2}}\right] .
\end{aligned}
$$

It depends on four arbitrary complex constants $\alpha, \beta, \gamma, \delta$.

The purpose of this article is twofold : (i) we first present a new, direct method, only based on the singularity structure of this ODE, in order to derive a first degree birational transformation for P6; (ii) we then establish for P6 the contiguity relation, interpreted as a discrete Painlevé equation.

A birational transformation is defined by

$$
u=r\left(U^{\prime}, U, X\right), U=R\left(u^{\prime}, u, x\right),
$$

with $r$ and $R$ rational functions, and it maps an equation

$$
E(u) \equiv \operatorname{Pn}(u, x, \boldsymbol{\alpha})=0, \boldsymbol{\alpha}=(\alpha, \beta, \gamma, \delta),
$$

into the same equation with different parameters

$$
E(U) \equiv \operatorname{Pn}(U, X, \boldsymbol{A})=0, \boldsymbol{A}=(A, B, \Gamma, \Delta),
$$

with some homography (usually the identity) between $x$ and $X$. The parameters $(\boldsymbol{\alpha}, \boldsymbol{A})$ must obey as many algebraic relations as elements in $\boldsymbol{\alpha}$. The degree of a birational transformation is by definition the highest degree in $U^{\prime}$ or $u^{\prime}$ of the numerator and the denominator of (1).

There are at least three uses of a birational transformation. The first one is an equivalence relation: two solutions exchanged by the transformation are considered as identical. The second use is to generate from a known solution a countable number of new solutions which may be of physical interest [3]. The third point of view, which we develop here, is the construction of a contiguity relation in order to define a nonautonomous discrete Painlevé equation.

The interest of being able to handle P6 is to avoid the tedious consideration of the other daughter Painlevé equations.

Indeed, all the similar results for these equations can be easily obtained by action of the confluence, among them all the first degree birational transformations of all Pn equations [5].

There currently exist two birational transformations of P6. Garnier [6, 7] was the first to find a birational transformation of $\mathrm{P} 6$, to establish a theorem of Schwarz on the problem of Plateau. This transformation, which has second degree, was later rediscovered by several authors [8, 9,10 . There also exists a first degree birational transformation, found by Okamoto [9] while studying the

Weyl group which preserves the Hamiltonian of P6, and rediscovered recently [1] in a different context. This transformation reads

$$
\begin{aligned}
\mathrm{T}_{6}: \frac{N}{u-U} & =\frac{x(x-1) U^{\prime}}{U(U-1)(U-x)}+\frac{\Theta_{0}}{U}+\frac{\Theta_{1}}{U-1}+\frac{\Theta_{x}-1}{U-x} \\
& =\frac{x(x-1) u^{\prime}}{u(u-1)(u-x)}+\frac{\theta_{0}}{u}+\frac{\theta_{1}}{u-1}+\frac{\theta_{x}-1}{u-x} \\
\theta_{j} & =\Theta_{j}-\frac{1}{2}\left(\sum \Theta_{k}\right)+\frac{1}{2}, j, k=\infty, 0,1, x, \\
\Theta_{j} & =\theta_{j}-\frac{1}{2}\left(\sum \theta_{k}\right)+\frac{1}{2}
\end{aligned}
$$


The transformation is clearly birational since the l.h.s. is homographic in both $u$ and $U$. In the above, the monodromy exponents $\boldsymbol{\theta}=\left(\theta_{\infty}, \theta_{0}, \theta_{1}, \theta_{x}\right)$ are defined as

$$
\theta_{\infty}^{2}=2 \alpha, \theta_{0}^{2}=-2 \beta, \theta_{1}^{2}=2 \gamma, \theta_{x}^{2}=1-2 \delta,
$$

and similarly for their uppercase counterparts, while the odd-parity constant $N$ takes the equivalent expressions

$$
\begin{aligned}
N & =\sum\left(\theta_{k}^{2}-\Theta_{k}^{2}\right) \\
& =1-\sum \Theta_{k}=-1+\sum \theta_{k} \\
& =2\left(\theta_{j}-\Theta_{j}\right), j=\infty, 0,1, x .
\end{aligned}
$$

The transformation of Garnier is an integer power [12] of this first degree transformation.

To achieve our goal (rely only on the singularity structure to find a birational transformation), we need to improve the singular manifold method so that it succeeds to obtain a birational transformation for P6. Originally introduced for partial differential equations (PDEs) by Weiss, Tabor and Carnevale [13], the singular manifold method is a powerful tool for deriving Bäcklund transformations, by considering only the singularity structure of the solutions. Its current achievements are detailed in summer school proceedings, see Refs. [14, 15]. An extension to ODEs has been proposed [16, 17, 18] to derive a birational transformation for the Painlevé equations, but its application to the master equation $\mathrm{P} 6$ is still an open problem. We solve it here by implementing an essential piece of information, which has up to now been overlooked.

The paper is organized as follows. In Section 2, we exploit the information that there always exists a homography between the derivative of the solution of the considered Painlevé equation Pn and the Riccati pseudopotential $Z$ introduced in the "truncation" assumption. This reduces the problem to finding two functions of two variables instead of two functions of three variables.

In Section 3, we implement this homography in the definition of a truncation, which allows us to overcome the major difficulty, coming, in the case of $\mathrm{P} 6$, from a residual coefficient which remains undetermined (technically coming from the value 1 of the Fuchs index). This difficulty is the main reason why P6 has never been handled before by methods based on the singularity structure. We also improve a previous conjecture [19] on the necessary form of the birational transformation. The truncation then becomes easy to solve and admits, up to the four homographies on $(U, x)$ which conserve $x$, a unique solution, which is the transformation $\mathrm{T}_{6}$ of Okamoto.

In Section 1 , we give the various representations of this transformation.

In Section 5, we solve the recurrence relation between the monodromy exponents of $\mathrm{P} 6$, and we build the contiguity relation, which defines a unique nonautonomous second order difference equation.

Finally, in Section 6, we propose a weaker definition of a Schlesinger transformation so as to identify this notion with that of birational transformation. 


\section{The fundamental homography}

From the result of Richard Fuchs [20], the P6 equation is obtained from the complete integrability of a linear differential system of order two. Therefore the pseudopotential of the singular manifold method has only one component $Z$ [21], which can be chosen so as to satisfy some Riccati ODE.

Each Pn equation which admits a birational transformation has one or several (four for P6) couples of families of movable simple poles with opposite residues $\pm u_{0}$, therefore both the onefamily truncation and the two-family truncation [22, 14, 15] are applicable. In the present paper, we consider only the one-family truncation, whose assumption is

$$
\begin{aligned}
& u=u_{0} Z^{-1}+U, u_{0} \neq 0, x=X, \\
& Z^{\prime}=1+z_{1} Z+z_{2} Z^{2}, z_{2} \neq 0,
\end{aligned}
$$

in which $u$ and $U$ satisfy (2) and (3), $\left(Z, z_{1}, z_{2}\right)$ are rational functions of $\left(x, U, U^{\prime}\right)$ to be determined. After this is done, the relation (12) represents half of the birational transformation.

Besides the equation (13), there exists a second Riccati equation in the present problem, this is the Painlevé equation (3) itself. Indeed, any $N$-th order, first degree ODE with the Painlevé property is necessarily [23, pp. 396-409] a Riccati equation for $U^{(N-1)}$, with coefficients depending on $x$ and the lower derivatives of $U$, in our case

$$
U^{\prime \prime}=A_{2}(U, x) U^{\prime 2}+A_{1}(U, x) U^{\prime}+A_{0}(U, x) .
$$

Since the group of invariance of a Riccati equation is the homographic group, the variables $U^{\prime}$ and $Z$ are linked by a homography, the three coefficients $g_{j}$ of which are rational in $(U, x)$. Let us define it as

$$
\left(U^{\prime}+g_{2}\right)\left(Z^{-1}-g_{1}\right)-g_{0}=0, g_{0} \neq 0 .
$$

This allows us to obtain the two coefficients $z_{j}$ of the Riccati pseudopotential equation (13) as explicit expressions of $\left(g_{j}, \partial_{U} g_{j}, \partial_{x} g_{j}, A_{2}, A_{1}, A_{0}, U^{\prime}\right)$. Indeed, eliminating $U^{\prime}$ between (14) and (15) defines a first order ODE for $Z$, whose identification with (13) modulo (15) provides three relations.

For the one-family truncation, these are

$$
\begin{aligned}
& g_{0}=g_{2}^{2} A_{2}-g_{2} A_{1}+A_{0}+\partial_{x} g_{2}-g_{2} \partial_{U} g_{2}, \\
& z_{1}=A_{1}-2 g_{1}+\partial_{U} g_{2}-\partial_{x} \log g_{0}+\left(2 A_{2}-\partial_{U} \log g_{0}\right) U^{\prime}, \\
& z_{2}=-g_{1} z_{1}-g_{1}^{2}-g_{0} A_{2}-\partial_{x} g_{1}-\left(\partial_{U} g_{1}\right) U^{\prime} .
\end{aligned}
$$

Therefore, the natural unknowns in the present problem are the two coefficients $g_{1}, g_{2}$ of the homography, which are functions of the two variables $(U, x)$, and not the two functions $\left(z_{1}, z_{2}\right)$ of the three variables $\left(U^{\prime}, U, x\right)$.

Remark. One must also consider the case when the relation between $Z^{-1}$ and $U^{\prime}$ is affine, excluded in (15). Assuming

$$
G_{1}\left(U^{\prime}+G_{2}\right)-Z^{-1}=0, G_{1} \neq 0
$$

the equation analogous to (16) is now

$$
\partial_{U} G_{1}+G_{1}^{2}+A_{2} G_{1}=0
$$

which for $\mathrm{P} 6$ admits no solution $G_{1}$ rational in $U$. 


\section{The truncation}

Just like the field $u$ is represented, see Eq. (12), by a Laurent series in $Z$ which terminates ("truncated series"), the l.h.s. $E(u)$ of the Pn equation (supposed written as a polynomial, i.e. without denominators involving $u$ ) can also be written as a truncated series in $Z$. This is achieved by the elimination of $u, Z^{\prime}, U^{\prime \prime}, U^{\prime}$ between (2), (3), (12), (13) and (15), followed by the elimination of $\left(g_{0}, z_{1}, z_{2}\right)$ from (16)-(18) ( $q$ denotes the singularity order of Pn written as a differential polynomial in $u$, it is -6 for $\mathrm{P} 6$ ),

$$
\begin{aligned}
& E(u)=\sum_{j=0}^{-q+2} E_{j}\left(U, x, u_{0}, g_{1}, g_{2}, \boldsymbol{\alpha}, \boldsymbol{A}\right) Z^{j+q-2}=0, \\
& \forall j: E_{j}\left(U, x, u_{0}, g_{1}, g_{2}, \boldsymbol{\alpha}, \boldsymbol{A}\right)=0 .
\end{aligned}
$$

The nonlinear determining equations $E_{j}=0$ are independent of $U^{\prime}$, and this is the main difference with previous work. In particular, in all cases successfully processed to date [17, 18], both $g_{1}$ and $2 A_{2}-\partial_{U} \log g_{0}$ vanish, which cancels the coefficient of $U^{\prime}$ in $z_{1}$ and $z_{2}$. In the case of P6, we are going to see that the truncation possesses a solution if and only if $\left(z_{1}, z_{2}\right)$ depends on $U^{\prime}$.

Another difference is the greater number $(-q+3$ instead of $-q+1)$ of equations $E_{j}=0$, which is due to the additional elimination of $U^{\prime}$ with (15).

The $-q+3$ determining equations (22) in the three unknown functions $u_{0}(x), g_{1}(U, x), g_{2}(U, x)$ (and the unknown scalars $\alpha, \beta, \gamma, \delta$ in terms of $A, B, \Gamma, \Delta)$ must be solved, as usual, by increasing values of their index $j$.

This truncation for P6 possesses a very nice invariance, which drastically helps the practical resolution. For any solution $g_{j}(U, x)$, there exist three other solutions, generated by the action on $g_{j}(U, x)$ of the four homographies of $U$ which conserve $x$ and $\mathrm{P} 6$, namely the identity and

$$
\begin{aligned}
& \mathrm{H}_{\text {badc }}: \boldsymbol{\theta}=\left(\begin{array}{llll}
0 & 1 & 0 & 0 \\
1 & 0 & 0 & 0 \\
0 & 0 & 0 & 1 \\
0 & 0 & 1 & 0
\end{array}\right) \boldsymbol{\Theta}, x=X, u=\frac{x}{U}, \\
& \mathrm{H}_{\text {dcba }}: \boldsymbol{\theta}=\left(\begin{array}{llll}
0 & 0 & 0 & 1 \\
0 & 0 & 1 & 0 \\
0 & 1 & 0 & 0 \\
1 & 0 & 0 & 0
\end{array}\right) \boldsymbol{\Theta}, x=X, u-x=\frac{x(x-1)}{U-x}, \\
& \mathrm{H}_{\text {cdab }}: \boldsymbol{\theta}=\left(\begin{array}{llll}
0 & 0 & 1 & 0 \\
0 & 0 & 0 & 1 \\
1 & 0 & 0 & 0 \\
0 & 1 & 0 & 0
\end{array}\right) \boldsymbol{\Theta}, x=X, u-1=\frac{1-x}{U-1}, \\
& \mathrm{H}_{\text {badc }}^{2}=\mathrm{H}_{\text {dcba }}^{2}=\mathrm{H}_{\text {cdab }}^{2}=1, \mathrm{H}_{\text {badc }} \mathrm{H}_{\mathrm{dcba}} \mathrm{H}_{\mathrm{cdab}}=1 .
\end{aligned}
$$

Let us now state a conjecture which will strongly restrict the coefficient $g_{2}$. From the expression of the direct half of the birational transformation,

$$
u=U+u_{0}\left(g_{1}(U, x)+\frac{g_{0}(U, x)}{U^{\prime}+g_{2}(U, x)}\right)
$$

the values of $U$ which correspond to $u=\infty$ are defined (apart from the poles of $g_{1}$ and $g_{0}$ ) by the ODE

$$
U^{\prime}+g_{2}(U, x)=0
$$

The point $u=\infty$ has the peculiarity to be a singular point of all the Pn equations and, for all known birational transformations of Pn (see the book [24 for P2 to P5, Ref. [7, formula (2.8)] and 
Ref. 9, p. 356] for P6), it happens that the ODE analogous to (28) is a Riccati ODE (or a product of Riccati ODEs in [7, 10]), i.e. the unique first order first degree ODE which has the Painlevé property. In at least one other example of higher order [25], the birational transformation between two different ODEs having the Painlevé property has a denominator which defines a P1 equation. Let us conjecture the generality of this property.

Conjecture. Given a birational transformation between two ODEs having the Painlevé property, for any singular point $u$ of the ODE, the ODE for $U$ defined by the direct half of the birational transformation has the Painlevé property.

This is an improvement of a previous conjecture by Fokas and Ablowitz 19, formula (2.6)] in two respects: both fields $u$ and $U$ are required to satisfy the same ODE, and no specific $U$-dependence is assumed for $g_{0}$ and $g_{1}$; their conjecture (whatever be $u$, Eq. (27) is a Riccati equation for $U$ ) happens to be true for P2-P5 but not for P6.

In the case of $\mathrm{P} 6$, our conjecture implies that the four expressions

$$
g_{2}, \frac{g_{0}}{U+u_{0} g_{1}}, \frac{g_{0}}{U-1+u_{0} g_{1}}, \frac{g_{0}}{U-x+u_{0} g_{1}},
$$

are second degree polynomials of $U$ with coefficients depending on $x$. One notices immediately, from the last three fractions, that there could exist a particular solution $g_{1}=0, g_{0}=g(x) U(U-1)(U-x)$. We are going to see that this is indeed the case.

The practical resolution of the truncation for P6 is performed in Appendix 8, and the coefficients of the fundamental homography are found to be

$$
\begin{aligned}
g_{0}= & \frac{N U(U-1)(U-x)}{u_{0} x(x-1)}, g_{1}=0, g_{2}=\frac{U(U-1)(U-x)}{x(x-1)}\left(\frac{\Theta_{0}}{U}+\frac{\Theta_{1}}{U-1}+\frac{\Theta_{x}-1}{U-x}\right), \\
u_{0}= & -\frac{x(x-1)}{\theta_{\infty}}, \theta_{\infty}=\frac{1}{2}\left(\Theta_{\infty}-\Theta_{0}-\Theta_{1}-\Theta_{x}+1\right), \\
N= & 1-\Theta_{\infty}-\Theta_{0}-\Theta_{1}-\Theta_{x}, \\
z_{1}= & \frac{1}{x(x-1)}\left(\left(\Theta_{1}+\Theta_{x}-1\right) U+\left(\Theta_{x}-1+\Theta_{0}\right)(U-1)+\left(\Theta_{0}+\Theta_{1}\right)(U-x)\right) \\
& +\frac{1}{x}+\frac{1}{x-1}, \\
z_{2}= & \frac{N \theta_{\infty}}{2(x(x-1))^{2}}((U-1)(U-x)+U(U-x)+U(U-1)) .
\end{aligned}
$$

Let us present this birational transformation in more detail.

\section{The elementary birational transformation of P6}

The eight signs $\left(s_{\infty}, s_{0}, s_{1}, s_{x}\right)$ and $\left(S_{\infty}, S_{0}, S_{1}, S_{x}\right)$, with $s_{j}^{2}=S_{j}^{2}=1$, of the monodromy exponents remain arbitrary and independent.

\subsection{The affine representation}

In the space of the monodromy exponents, the direct birational transformation and its inverse have an affine representation

$$
\boldsymbol{\theta}=M_{1} \boldsymbol{\Theta}+M_{0}, \boldsymbol{\theta}=\left(\begin{array}{c}
\theta_{\infty} \\
\theta_{0} \\
\theta_{1} \\
\theta_{x}
\end{array}\right), \boldsymbol{\Theta}=\left(\begin{array}{c}
\Theta_{\infty} \\
\Theta_{0} \\
\Theta_{1} \\
\Theta_{x}
\end{array}\right)
$$


in which $M_{1}$ and $M_{0}$ are matrices of rational numbers. These are

$$
\begin{gathered}
\mathrm{T}_{6}:\left(\begin{array}{c}
s_{\infty} \theta_{\infty} \\
s_{0} \theta_{0} \\
s_{1} \theta_{1} \\
s_{x} \theta_{x}
\end{array}\right)=\frac{1}{2}\left(\begin{array}{cccc}
1 & -1 & -1 & -1 \\
-1 & 1 & -1 & -1 \\
-1 & -1 & 1 & -1 \\
-1 & -1 & -1 & 1
\end{array}\right)\left(\begin{array}{c}
S_{\infty} \Theta_{\infty} \\
S_{0} \Theta_{0} \\
S_{1} \Theta_{1} \\
S_{x} \Theta_{x}
\end{array}\right)+\frac{1}{2}\left(\begin{array}{l}
1 \\
1 \\
1 \\
1
\end{array}\right), \\
\mathrm{T}_{6}^{-1}:\left(\begin{array}{c}
S_{\infty} \Theta_{\infty} \\
S_{0} \Theta_{0} \\
S_{1} \Theta_{1} \\
S_{x} \Theta_{x}
\end{array}\right)=\frac{1}{2}\left(\begin{array}{cccc}
1 & -1 & -1 & -1 \\
-1 & 1 & -1 & -1 \\
-1 & -1 & 1 & -1 \\
-1 & -1 & -1 & 1
\end{array}\right)\left(\begin{array}{c}
s_{\infty} \theta_{\infty} \\
s_{0} \theta_{0} \\
s_{1} \theta_{1} \\
s_{x} \theta_{x}
\end{array}\right)+\frac{1}{2}\left(\begin{array}{l}
1 \\
1 \\
1 \\
1
\end{array}\right),
\end{gathered}
$$

and, depending on the signs, one has $M_{1}^{n}=1$, with $n=2,3,4$ or 6 . The convention adopted for the signs is aimed at making (6) equal to its inverse when all signs are +1 .

\subsection{The birational representation}

Denoting $N$ the odd-parity constant

$$
\begin{aligned}
N & =\sum\left(\theta_{k}^{2}-\Theta_{k}^{2}\right) \\
& =1-\sum S_{k} \Theta_{k}=-1+\sum s_{k} \theta_{k} \\
& =2\left(s_{j} \theta_{j}-S_{j} \Theta_{j}\right), j=\infty, 0,1, x,
\end{aligned}
$$

the four algebraic relations between $\alpha, \beta, \gamma, \delta$ and $A, B, \Gamma, \Delta$ are

$$
\forall j=\infty, 0,1, x:\left(\theta_{j}^{2}+\Theta_{j}^{2}-(N / 2)^{2}\right)^{2}-\left(2 \theta_{j} \Theta_{j}\right)^{2}=0,
$$

equivalent to the affine representation (6). Two sets of expressions are appropriate to represent the birational transformation, depending on its later use.

The first set involves only the squares of the monodromy exponents,

$$
\begin{aligned}
\frac{N}{u-U}=\frac{x(x-1) U^{\prime}}{U(U-1)(U-x)} & +\left(\frac{\theta_{0}^{2}-\Theta_{0}^{2}}{N}-\frac{N}{4}\right) \frac{1}{U}+\left(\frac{\theta_{1}^{2}-\Theta_{1}^{2}}{N}-\frac{N}{4}\right) \frac{1}{U-1} \\
& +\left(\frac{\theta_{x}^{2}-\Theta_{x}^{2}}{N}-\frac{N}{4}-1\right) \frac{1}{U-x} \\
=\frac{x(x-1) u^{\prime}}{u(u-1)(u-x)} & +\left(\frac{\theta_{0}^{2}-\Theta_{0}^{2}}{N}+\frac{N}{4}\right) \frac{1}{u}+\left(\frac{\theta_{1}^{2}-\Theta_{1}^{2}}{N}+\frac{N}{4}\right) \frac{1}{u-1} \\
& +\left(\frac{\theta_{x}^{2}-\Theta_{x}^{2}}{N}+\frac{N}{4}-1\right) \frac{1}{u-x} .
\end{aligned}
$$

The second set of expressions, affine in the eight signed monodromy exponents,

$$
\begin{aligned}
\frac{N}{u-U} & =\frac{x(x-1) U^{\prime}}{U(U-1)(U-x)}+\frac{S_{0} \Theta_{0}}{U}+\frac{S_{1} \Theta_{1}}{U-1}+\frac{S_{x} \Theta_{x}-1}{U-x} \\
& =\frac{x(x-1) u^{\prime}}{u(u-1)(u-x)}+\frac{s_{0} \theta_{0}}{u}+\frac{s_{1} \theta_{1}}{u-1}+\frac{s_{x} \theta_{x}-1}{u-x},
\end{aligned}
$$

is adapted to the construction of the contiguity relation, which is done in Section .

Both sets allow one to directly apply the well known degeneracy (Ref. [2] in the space $\boldsymbol{\alpha}$, Ref. [26] in the space $\boldsymbol{\theta}$ ) to generate birational transformations of the other Pn equations. This is done in a forthcoming paper [5].

\section{Contiguity relation}

From each birational transformation, one easily deduces a contiguity relation, which generalizes, as noted by Garnier [7], that of the hypergeometric equation of Gauss. Its systematic computation is as follows 沺. 
1. Consider the birational transformation, i.e. the direct birational transformation and its inverse

$$
\begin{aligned}
& u=f\left(U, U^{\prime}, x, \boldsymbol{\theta}, \boldsymbol{\Theta}\right), \boldsymbol{\theta}=g(\boldsymbol{\Theta}), \\
& U=F\left(u, u^{\prime}, x, \boldsymbol{\Theta}, \boldsymbol{\theta}\right), \boldsymbol{\Theta}=G(\boldsymbol{\theta}) .
\end{aligned}
$$

2. Evaluate it at the values $(\underline{v}, v, \bar{v})$ taken by a discrete variable at three contiguous points $(z-h, z, z+h)$, with $z=n h$,

$$
\begin{aligned}
\bar{v} & =f\left(v, v^{\prime}, x, f(\boldsymbol{\theta}), \boldsymbol{\theta}\right), \\
\underline{v} & =F\left(v, v^{\prime}, x, F(\boldsymbol{\theta}), \boldsymbol{\theta}\right) .
\end{aligned}
$$

3. Eliminate the variable $v^{\prime}$ between these two relations,

$$
G(\bar{v}, \underline{v}, v, x, \boldsymbol{\theta})=0 .
$$

For P6, equations (46)-(47) are equivalent to

$$
\begin{aligned}
& \frac{x(x-1) U^{\prime}}{U(U-1)(U-x)}=2 \frac{s_{j} \theta_{j}-S_{j} \Theta_{j}}{u-U}-\left(\frac{S_{0} \Theta_{0}}{U}+\frac{S_{1} \Theta_{1}}{U-1}+\frac{S_{x} \Theta_{x}-1}{U-x}\right), \\
& \frac{x(x-1) u^{\prime}}{u(u-1)(u-x)}=-2 \frac{S_{j} \Theta_{j}-s_{j} \theta_{j}}{u-U}-\left(\frac{s_{0} \theta_{0}}{u}+\frac{s_{1} \theta_{1}}{u-1}+\frac{s_{x} \theta_{x}-1}{u-x}\right),
\end{aligned}
$$

in which $j$ is anyone of the four singular points $(\infty, 0,1, x)$, and the contiguity relation is

$$
\begin{aligned}
& \frac{\varphi(n+1 / 2)}{\bar{v}-v}+\frac{\varphi(n-1 / 2)}{\underline{v}-v}=\frac{s_{0} \theta_{0}-S_{0} \Theta_{0}}{v}+\frac{s_{1} \theta_{1}-S_{1} \Theta_{1}}{v-1}+\frac{s_{x} \theta_{x}-S_{x} \Theta_{x}}{v-x}, \\
& \varphi(n)=\frac{1}{2}\left(s_{\infty} \theta_{\infty}+s_{0} \theta_{0}+s_{1} \theta_{1}+s_{x} \theta_{x}-1\right),
\end{aligned}
$$

in which $\boldsymbol{\theta}$ is taken at the center point $z=z_{0}+n h$. This very simple expression is clearly invariant under any permutation of the four singular points of $\mathrm{P} 6$.

This contiguity relation (53) can be interpreted as a second order discrete equation [4]. The two-point recurrence relation (6) admits five classes of solutions. Each class, characterized by a signature, leads to a different contiguity relation (53), i.e. to a different second order discrete equation. Four of them are autonomous (signatures $\left(s_{j} S_{j}\right)=(---+),(--++),(-+++)$, $(++++))$, they cannot admit a continuum limit to a Painlevé equation. The only nonautonomous one (signature $(----))$ is

$$
\begin{aligned}
& \frac{n+1 / 2}{\bar{v}-v}+\frac{n-1 / 2}{\underline{v}-v}=\frac{n+K_{2}(-1)^{n}}{v}+\frac{n+K_{3}(-1)^{n}}{v-1}+\frac{n+K_{4}(-1)^{n}}{v-x}, \\
& K_{2}=-k_{2}+k_{3}+k_{4}, K_{3}=k_{2}-k_{3}+k_{4}, K_{4}=k_{2}+k_{3}-k_{4} .
\end{aligned}
$$

In the continuum limit, among the six simple poles of $v$ in the sum (including $\infty$ ), the first two will create a second order derivative and the four others will define at most four singular points. Since none of the last four poles depends on $n$, it is impossible that the continuum limit be P6.

The transform of this discrete equation under

$$
(\bar{v}, v, \underline{v}) \mapsto(\bar{v}, x / v, \underline{v})
$$

has already been obtained [27, Eq. (1.5)] as a reduction of a lattice KdV equation, together with a discrete Lax pair and a continuum limit to the full P5. This is in agreement with the continuum limit of the hypergeometric contiguity relation, which is not the hypergeometric equation but a confluent one. Nevertheless, we do not know of a general proof of this feature. 


\section{$6 \quad$ On Schlesinger transformations}

Up to this point we have carefully avoided using the expression Schlesinger transformation, and this is because of some discrepancy which we would want firstly, to point out, secondly, to try to correct.

The relevant items are

1. a birational transformation of some nonlinear ODE, whose definition presents no ambiguity,

2. the monodromy data, also unambiguously defined by the isomonodromic deformation of some linear ODE,

3. a Schlesinger transformation, whose present definition, recalled below, is in our opinion unsatisfactory.

A Schlesinger transformation (ST) was originally defined [28] as a discrete transformation preserving the monodromy of a given linear ODE, and Schlesinger explicitly prescribed [29, p. 136] [28, p. 134] that monodromy exponents $\theta$ should be shifted by integer values, which is a sufficient condition to preserve the monodromy data. All subsequent authors, in particular Garnier [7] and Jimbo and Miwa 30 (who created the expression Schlesinger transformation) complied with this prescription because all had in mind the isomonodromic deformations.

The discrepancy is the following. It would be nice to have a one-to-one correspondence, hence an equivalence, between the notion of birational transformation and that of Schlesinger transformation. With the present definition, this is not the case for the birational transformation (42), whose associated affine transformation (6) -(7) does not obey the prescription of Schlesinger of shifts by integers and therefore does not preserve the monodromy data. A first step in this direction was recently made in Refs. [31, 32, 33], who relaxed the prescription to shifts by half-integers, so as to globally change the monodromy matrix to its opposite rather than to conserve it.

What happens here is one step beyond: the monodromy matrix is changed to its opposite, but the shifts by half-integers occur not in the space of monodromy exponents, but in the space adapted to the affine Weyl group $\mathrm{D}_{4}$ of P6. Indeed, in the basis of Okamoto (58),

$$
b_{1}=\left(\theta_{0}+\theta_{1}\right) / 2, b_{2}=\left(\theta_{0}-\theta_{1}\right) / 2, b_{3}=\left(\theta_{x}-1-\theta_{\infty}\right) / 2, b_{4}=\left(\theta_{x}-1+\theta_{\infty}\right) / 2,
$$

the affine representation (6) becomes (when all signs are chosen equal to +1 to simplify)

$$
\mathrm{T}_{6}:\left(\begin{array}{l}
b_{1} \\
b_{2} \\
b_{3} \\
b_{4}
\end{array}\right)=\left(\begin{array}{c}
-B_{3} \\
B_{2} \\
-B_{1} \\
B_{4}
\end{array}\right) .
$$

In the monodromy matrix, the set of four trigonometric lines in Eq. (3.19) of Ref. [10] just undergoes a global sign change.

To conclude this discussion, in order to identify Schlesinger transformation and birational transformation, at least for P6 (we leave the full generality to mathematicians), we propose that a Schlesinger transformation be defined as any discrete transformation which either conserves or changes to its opposite the monodromy matrix, without any additional prescription on the monodromy exponents.

As a consequence of this weaker definition, the matrices $M_{1}$ and $M_{0}$ in (35) will have halfinteger elements. Another consequence of practical importance is the identification, at least for the Pn equations, of the two notions Schlesinger transformation, birational transformation, since they will now have a one-to-one correspondence. 


\section{Conclusion}

The fundamental homography between the derivative of the solution of the Painlevé equation and the Riccati pseudopotential has allowed us to define a consistent truncation. The result thus obtained for $\mathrm{P} 6$ is a first degree birational transformation, and its contiguity relation defines a single nonautonomous second order difference equation. Its degeneracies under the confluence of the Pn equations could provide new second order discrete equations.

As an application, one can iterate this birational transformation, starting from the two-parameter solution which Picard established for $\theta_{j}^{(0)}=0, j=\infty, 0,1, x$, to carry it to any $\boldsymbol{\theta}$ such that $2 \theta_{j}$ and $\sum \theta_{j}$ be arbitrary integers. Any iterate will be some algebraic transform of the solution of Picard.

Two important open problems remain in the domain of truncations, namely to define truncations able to provide, firstly a Lax pair of P6, secondly a Lax pair for the discrete equations built from the Schlesinger transformation.

\section{Acknowledgments}

The authors acknowledge the financial support of the Tournesol grant T99/040. MM acknowledges the financial support of the IUAP Contract No. P4/08 funded by the Belgian government and the support of CEA.

\section{Appendix. One-family truncation for $\mathrm{P} 6$}

All families of movable singularities are equivalent under homographies. The data for this unique representative family are

$$
q=-6, u_{0}=-\frac{x(x-1)}{\theta_{\infty}}, \text { Fuchs index } 1,
$$

so there are nine determining equations (22) $E_{j}=0, j=0, \ldots,-q+2$.

The three coefficients $\left(A_{2}, A_{1}, A_{0}\right)$ are defined by (14),

$$
\begin{aligned}
& A_{2}=\frac{1}{2}\left[\frac{1}{U}+\frac{1}{U-1}+\frac{1}{U-x}\right], A_{1}=-\left[\frac{1}{x}+\frac{1}{x-1}+\frac{1}{U-x}\right], \\
& A_{0}=\frac{U(U-1)(U-x)}{x^{2}(x-1)^{2}}\left[A+B \frac{x}{U^{2}}+\Gamma \frac{x-1}{(U-1)^{2}}+\Delta \frac{x(x-1)}{(U-x)^{2}}\right] .
\end{aligned}
$$

Equation $j=0$ only depends on $u_{0}$ and its solution is (60). Equation $j=1$ is identically satisfied, as a consequence of the value 1 of the Fuchs index. There remain seven equations (22) $j=2, \ldots, 8$, in the two unknowns $g_{k}(U, x), k=1,2$. The next two ones are

$$
\begin{aligned}
E_{2} \equiv & \left(g_{2} \partial_{U}-\partial_{x}\right) g_{1}+g_{1}^{2}+g_{0}^{-1} F_{0} g_{1}+g_{0}^{-2} F_{1}=0, \\
E_{3} \equiv & \left(-g_{2}^{2} \partial_{U}^{2}+2 g_{2} \partial_{U} \partial_{x}-\partial_{x}^{2}\right) g_{1}+\left(-g_{2}^{2} A_{2}+g_{2} A_{1}-A_{0}-3 g_{0}\right) \partial_{U} g_{1} \\
& +2 g_{1}^{3}+g_{0}^{-1} F_{2} g_{1}^{2}+g_{0}^{-2} F_{3} g_{1}+g_{0}^{-3} F_{4}=0,
\end{aligned}
$$

in which the functions $F_{k}$ are differential polynomials of $\left(g_{0}, g_{2}, A_{2}, A_{1}, A_{0}\right)$,

$$
\begin{aligned}
& F_{0} \equiv\left(2 A_{2} g_{2}-A_{1}+\theta_{\infty} \frac{2+2 x-6 U}{3 x(x-1)}\right) g_{0}+\partial_{x} g_{0}-\partial_{U}\left(g_{0} g_{2}\right), \\
& F_{1} \equiv 48 \text { terms. }
\end{aligned}
$$

According to the conjecture of section 3 , let us assume that $g_{2}$ is a second degree polynomial of $U$, conveniently defined as

$$
g_{2}=U(U-1)(U-x)\left(\frac{f_{0}(x)}{U}+\frac{f_{1}(x)}{U-1}+\frac{f_{x}(x)-1}{U-x}\right) .
$$


To take full advantage of the symmetry of $\mathrm{P} 6$, it is useful to also define

$$
f_{\infty}=1-f_{0}-f_{1}-f_{1}
$$

Equation (16) then provides

$$
\begin{aligned}
g_{0}= & \frac{1}{2(x(x-1))^{2}}\left\{\left(\Theta_{\infty}^{2}-f_{\infty}^{2}\right) U(U-1)(U-x)-\left(\Theta_{0}^{2}-f_{0}^{2}\right) \frac{x(U-1)(U-x)}{U}\right. \\
& \left.+\left(\Theta_{1}^{2}-f_{1}^{2}\right) \frac{(x-1) U(U-x)}{U-1}-\left(\Theta_{x}^{2}-f_{x}^{2}\right) \frac{x(x-1) U(U-1)}{U-x}\right\} \\
& +\frac{1}{x(x-1)}\left(f_{0}^{\prime}(U-1)(U-x)+f_{1}^{\prime} U(U-x)+f_{x}^{\prime} U(U-1)\right) .
\end{aligned}
$$

At this point, one could enforce the second part of the conjecture, i.e. solve the three diophantine conditions on the rational functions $g_{0}$ and $g_{1}$ of $U$. Let us instead proceed with the truncation.

Equation $j=2$ first provides the degrees in $U$ of the numerator and denominator of the rational function $g_{1}$. Indeed, the degrees in $U$ of the polynomial coefficients of $E_{2}$ are

$$
\text { degree }(U(U-1)(U-x))^{2} F_{0}=10, \text { degree }(U(U-1)(U-x))^{4} F_{1}=20,
$$

therefore $U(U-1)(U-x) g_{1}$ is a fourth degree polynomial of $U$, whose convenient definition implementing the invariance of $\mathrm{P} 6$ is

$$
g_{1}=\Psi_{\infty} \frac{U}{x(x-1)}+\frac{\Psi_{0}}{(x-1) U}-\frac{\Psi_{1}}{x(U-1)}+\frac{\Psi_{x}}{U-x}+\frac{\Psi_{c}}{x(x-1)},
$$

in which the five functions $\Psi_{k}$ only depend on $x$. Equation $j=2$ then becomes a polynomial in $U$, equivalently expanded in powers of $1 / U, U, U-1$, or $U-x$,

$$
\begin{aligned}
& E_{2} \equiv \sum_{k=0}^{20} E_{2, k}^{(\infty)} U^{20-k} \equiv \sum_{k=0}^{20} E_{2, k}^{(0)} U^{k} \equiv \sum_{k=0}^{20} E_{2, k}^{(1)}(U-1)^{k} \equiv \sum_{k=0}^{20} E_{2, k}^{(x)}(U-x)^{k}=0, \\
& \forall i \in\{\infty, 0,1, x\}: E_{2, k}^{(i)}=0 .
\end{aligned}
$$

The four equations $k=0$ factorize,

$$
j=2, k=0: \begin{cases}\left(\Theta_{\infty}^{2}-f_{\infty}^{2}\right) & \left(\Theta_{\infty}^{2}-\left(f_{\infty}+2 \Psi_{\infty}-2 \theta_{\infty}\right)^{2}\right)=0 \\ \left(\Theta_{0}^{2}-f_{0}^{2}\right) & \left(\Theta_{0}^{2}-\left(f_{0}+2 \Psi_{0}\right)^{2}\right)=0 \\ \left(\Theta_{1}^{2}-f_{1}^{2}\right) & \left(\Theta_{1}^{2}-\left(f_{1}+2 \Psi_{1}\right)^{2}\right)=0 \\ \left(\Theta_{x}^{2}-f_{x}^{2}\right) & \left(\Theta_{x}^{2}-\left(f_{x}+2 \Psi_{x}\right)^{2}\right)=0\end{cases}
$$

thus defining five possibilities since the signs of $\Theta_{j}$ are not prescribed.

The first possibility $\forall i \in\{\infty, 0,1, x\}: \Theta_{i}^{2}-f_{i}^{2}=0$ is ruled out since the $\Theta_{i}$ 's must remain arbitrary.

The second possibility, that one $\Theta_{i}^{2}-f_{i}^{2}$ be nonzero and the three others vanish, defines four equivalent subcases, e.g.

$$
f_{0}=\Theta_{0}, f_{1}=\Theta_{1}, f_{x}=\Theta_{x}, \Psi_{\infty}=\theta_{\infty}-\frac{1}{2}\left(\Theta_{\infty}-\Theta_{0}-\Theta_{1}-\Theta_{x}+1\right) .
$$

At $j=2, k=4$, one obtains

$$
j=2, k=4: \Psi_{c}=-\frac{x+1}{3} \Psi_{\infty}, \Psi_{0}\left(\Psi_{0}-\Theta_{0}\right)=0, \Psi_{1}\left(\Psi_{1}-\Theta_{1}\right)=0, \Psi_{x}\left(\Psi_{x}-\Theta_{x}\right)=0 .
$$

At $j=2, k=5$, the subcase $\left(\Psi_{0}-\Theta_{0}\right)\left(\Psi_{1}-\Theta_{1}\right)\left(\Psi_{x}-\Theta_{x}\right)=0$ is ruled out and one obtains

$$
\begin{aligned}
& 12 \theta_{0}^{2}-4 \theta_{\infty}^{2}=3\left(-\Theta_{\infty}+\Theta_{0}-\Theta_{1}-\Theta_{x}+1\right)^{2}-\left(\Theta_{\infty}-\Theta_{0}-\Theta_{1}-\Theta_{x}+1\right)^{2}, \\
& 12 \theta_{1}^{2}-4 \theta_{\infty}^{2}=3\left(-\Theta_{\infty}-\Theta_{0}+\Theta_{1}-\Theta_{x}+1\right)^{2}-\left(\Theta_{\infty}-\Theta_{0}-\Theta_{1}-\Theta_{x}+1\right)^{2}, \\
& 12 \theta_{x}^{2}-4 \theta_{\infty}^{2}=3\left(-\Theta_{\infty}-\Theta_{0}-\Theta_{1}+\Theta_{x}+1\right)^{2}-\left(\Theta_{\infty}-\Theta_{0}-\Theta_{1}-\Theta_{x}+1\right)^{2} .
\end{aligned}
$$


This exhausts the equation $j=2$. Then, the equation $j=3$ is a polynomial of degree 3 in $U$, and the equation $E_{3, k}^{(0)}=0$ provides the last information,

$$
\theta_{\infty}=\frac{1}{2}\left(\Theta_{\infty}-\Theta_{0}-\Theta_{1}-\Theta_{x}+1\right) .
$$

Therefore, this second possibility (one $\Theta_{i}^{2}-f_{i}^{2}$ nonzero and the three others equal to zero) defines the solution

$$
g_{0}=\frac{N U(U-1)(U-x)}{u_{0} x(x-1)}, g_{1}=0, g_{2}=\left(\frac{\Theta_{0}}{U}+\frac{\Theta_{1}}{U-1}+\frac{\Theta_{x}-1}{U-x}\right) \frac{g_{0} u_{0}}{N}, u_{0}=-\frac{x(x-1)}{\theta_{\infty}} .
$$

With the three choices other than (74), one would simply obtain the three solutions deduced from that one by applying to the r.h.s. of $(27)$ the homographies $\mathrm{H}_{\mathrm{badc}}, \mathrm{H}_{\mathrm{dcba}}, \mathrm{H}_{\mathrm{cdab}}$, solutions characterized by the following values of $g_{1}$,

$$
u_{0} g_{1}=0, \frac{x}{U}-U, x+\frac{x(x-1)}{U-x}-U, 1+\frac{1-x}{U-1}-U .
$$

The third, fourth and fifth possibilities of vanishing of the four $\Theta_{i}^{2}-f_{i}^{2}$ are currently under examination, just to be sure that they provide no other solution.

The full results are given in the text.

\section{References}

[1] B. Gambier, Sur les équations différentielles du second ordre et du premier degré dont l'intégrale générale est à points critiques fixes, Thèse, Paris (1909); Acta Math. 33 (1910) $1-55$.

[2] P. Painlevé, Sur les équations différentielles du second ordre à points critiques fixes, C. R. Acad. Sc. Paris 143 (1906) 1111-1117.

[3] M. Mazzocco, Rational solutions of the Painlevé-VI equation, J. Phys. A 34 (2001) 2281-2294. nlin.SI/0007036.

[4] A. S. Fokas, B. Grammaticos, and A. Ramani, From continuous to discrete Painlevé equations, Journal of mathematical analysis and applications 180 (1993) 342-360.

[5] R. Conte and M. Musette, First degree birational transformations of the Painlevé equations and their contiguity relations, J. Phys. A 34 (2001) 10507-10522. nlin.SI/0110028.

[6] R. Garnier, Sur l'existence de relations entre des fonctions contiguës de Painlevé, C. R. Acad. Sc. Paris 217 (1943) 60-62.

[7] R. Garnier, Sur un théorème de Schwarz, Comm. Math. Helv. 25 (1951) 140-172.

[8] A. S. Fokas and Y. C. Yortsos, The transformation properties of the sixth Painlevé equation and one-parameter families of solutions, Lett. Nuovo Cimento 30 (1981) 539-544.

[9] K. Okamoto, Studies on the Painlevé equations, I, Sixth Painlevé equation, Ann. Mat. Pura Appl. 146 (1987) 337-381.

[10] U. Muğan and A. Sakka, Schlesinger transformations for Painlevé VI equation, J. Math. Phys. 36 (1995) 1284-1298.

[11] F. W. Nijhoff, N. Joshi, and A. Hone, On the discrete and continuous Miura chain associated with the sixth Painlevé equation, Phys. Lett. A 264 (2000) 396-406. 
[12] R. Conte, Sur les transformations de Schlesinger de la sixième équation de Painlevé, C. R. Acad. Sc. Paris 332 (2001) 501-504. math.CA/0103165.

[13] J. Weiss, M. Tabor, and G. Carnevale, The Painlevé property for partial differential equations, J. Math. Phys. 24 (1983) 522-526.

[14] M. Musette, Painlevé analysis for nonlinear partial differential equations, The Painlevé property, one century later, 517-572, ed. R. Conte, CRM series in mathematical physics (Springer, New York, 1999).

[15] R. Conte, Exact solutions of nonlinear partial differential equations by singularity analysis, Direct and inverse methods in nonlinear evolution equations, 83 pages, ed. A. Greco (Springer, Berlin, 2001). nlin-si/0009024. CIME school, Cetraro, 5-12 September 1999.

[16] P. A. Clarkson, N. Joshi, and A. Pickering, Bäcklund transformations for the second Painlevé hierarchy: a modified truncation approach, Inverse Problems 15 (1999) 175-187.

[17] P. Gordoa, N. Joshi, and A. Pickering, Mappings preserving locations of movable poles: a new extension of the truncation method to ordinary differential equations, Nonlinearity 12 (1999) 955-968.

[18] P. Gordoa, N. Joshi, and A. Pickering, Mappings preserving locations of movable poles: II. The third and fifth Painlevé equations, Nonlinearity 14 (2001) 567-582.

[19] A. S. Fokas and M. J. Ablowitz, On a unified approach to transformations and elementary solutions of Painlevé equations, J. Math. Phys. 23 (1982) 2033-2042.

[20] R. Fuchs, Sur quelques équations différentielles linéaires du second ordre, C. R. Acad. Sc. Paris 141 (1905) 555-558.

[21] M. Musette and R. Conte, Algorithmic method for deriving Lax pairs from the invariant Painlevé analysis of nonlinear partial differential equations, J. Math. Phys. 32 (1991) 14501457.

[22] M. Musette and R. Conte, The two-singular manifold method, I. Modified KdV and sineGordon equations, J. Phys. A 27 (1994) 3895-3913.

[23] P. Painlevé, Leçons sur la théorie analytique des équations différentielles (Leçons de Stockholm, 1895) (Hermann, Paris, 1897). Reprinted, Oeuvres de Paul Painlevé, vol. I (Éditions du CNRS, Paris, 1973).

[24] V. I. Gromak and N. A. Lukashevich, The analytic solutions of the Painlevé equations, 157 pages (in Russian), (Universitetskoye Publishers, Minsk, 1990). ISBN 5-7855-0319-0.

[25] A. N. W. Hone, Nonautonomous Hénon-Heiles systems, Physica D 118 (1998) 1-16. Bolvint/9703005.

[26] K. Okamoto, Isomonodromic deformation and Painlevé equations, and the Garnier system, J. Fac. Sci. Univ. Tokyo, Sect. IA 33 (1986) 575-618.

[27] F. W. Nijhoff, A. Ramani, B. Grammaticos, and Y. Ohta, On discrete Painlevé equations associated with the lattice KdV systems and the Painlevé VI equation, Stud. Appl. Math. 106 (2001) 261-314. solv-int/9812011.

[28] L. Schlesinger, Über eine Klasse von Differentialsystemen beliebiger Ordnung mit festen kritischen Punkten, J. für r. und angew. Math. 141 (1912) 96-145. 
[29] L. Schlesinger, Vorlesungen über lineare Differentialgleichungen (Teubner, Leipzig und Berlin, 1908).

[30] M. Jimbo and T. Miwa, Monodromy preserving deformations of linear ordinary differential equations with rational coefficients. II, Physica D 2 (1981) 407-448.

[31] P. Deift, A. R. Its, A. Kapaev, and X. Zhou, On the algebro-geometric integration of the Schlesinger equations, Commun. Math. Phys. 203 (1999) 613-633.

[32] A. V. Kitaev and D. A. Korotkin, On solutions of the Schlesinger equations in terms of $\Theta$-functions, 24 pages, preprint AEI-052 (January 1998). math-ph/9810007.

[33] D. A. Korotkin, N. Manojlović, and H. Samtleben, Schlesinger transformations for elliptic isomonodromic deformations, J. Math. Phys. 41 (2000) 3125-3141. 